\title{
The political economy of rationing in social health insurance *
}

\author{
Friedrich Breyer \\ Fakultät für Wirtschaftswissenschaften und Statistik, Universität Konstanz, Postfach 5560 D 135, \\ D-78434 Konstanz, Germany
}

\begin{abstract}
Due to the rapid progress in medical technology social insurance systems will soon no longer be able to grant health services without limits but must employ non-price rationing devices. This raises the question how these limits will be determined. Here we consider a direct democracy where the size of the social health insurance plan is determined in a popular referendum using simple majority rule. Moreover, two different kinds of rationing are distinguished according to whether additional private purchases of health care are allowed. For both systems we examine the size of the social insurance system in a political equilibrium, and we compare the results in particular with respect to their distributional effects.
\end{abstract}

\section{Introduction}

In some of the richest industrial nations of the western hemisphere, the institution of a social (health) insurance within the last century has helped to bring about a considerable degree of equality in the distribution of health care services. Since this contributed to a tremendous increase in life expectancy, it is with some justification regarded as a major achievement of modern societies. At least in this respect, the so-called "social" market economies have simultaneously reached a continuous improvement as well as some equality in the well-being of all members of society. Wealth and poverty are responsible for different consumption possibilities, but no longer for the survival chances as such. Life-prolonging health services are not allocated according to the willingness and ability to pay but almost exclusively according to criteria of medical "need".

The overall wealth has enabled these privileged societies to withhold the resources needed to extend human life and enhance its quality from the usual

\footnotetext{
* Funding for this research from the Norwegian Research Council under the Ruhrgas Scholarship Scheme and valuable comments by two anonymous referees are gratefully acknowledged.
} 
struggle for distributive shares. Fundamental norms of solidarity, which were previously considered as unachievable ideals, have to a large extent been realized in the institutions of social insurance in general and the health care system in particular. At the same time, it did not seem necessary to introduce explicit non-price rationing methods since all services demanded at zero price could at least in principle be supplied by the health care providers and financed through payroll taxes.

Today it is certain that this happy era of at most marginal inequality in the provision of health services has already ended or must soon do so. Due to the rapid progress in medical technology an allocation of health services unrelated to willingness or ability to pay and yet free of non-price barriers to consumption will no longer be feasible. According to the opinion of health care experts the industrial countries will very soon reach a point where their entire national product could be (effectively!) spent on health services. This means that access to health services can not be granted without limits but must be rationed. This would be true even if the supply free of charge could be restricted to those services that would undoubtedly yield positive utility in terms of lengthening the recipient's life or at least improving his well-being.

Therefore, even fundamental survival chances - as far as they can be influenced by health care consumption - will eventually have to be distributed by some rationing device. Since this problem will be "solved" by the institutional arrangements of the health system in any event, it is consistent with the spirit of democratic societies to solve it with the use of some explicit rationing method rather than implicitly.

The normative problems involved in the different possible types of rationing have been intensively discussed elsewhere (see Breyer and Kliemt 1994 and the literature quoted therein). The present paper addresses the positive aspect of the problem. It starts from the assumption that in a democratic society any type of rationing has to be accepted by the majority of voters. Thus I leave the paradigm of a "benevolent dictator" usually employed in normative analysis and assume instead that explicit democratic decisions are taken on the size and composition of the "health care budget", i.e. the services that will be provided through social health insurance to every member of society free of charge. Furthermore, to simplify the analysis, I shall consider a direct democracy where the size of the social health insurance plan is determined in a popular referendum using simple majority rule.

However, I shall distinguish two different kinds of rationing: "strong rationing" means that no member of society will be allowed to consume additional amounts of health (insurance) services beyond those provided within the social insurance system. Moreover, I shall assume that this rule, which is supported by many as ensuring "equality before death", can be effectively enforced ${ }^{2}$. "Weak rationing", on the other hand, means that such additional consumption is legal. It is plausible that the availability of additional services will have an effect on the optimum size of the social insurance system desired by voters, and - considering the redistributive feature of the social insurance system - that this effect will differ across income groups. Therefore I shall, after introducing the model in Sect. 2 , examine both cases separately (in Sects. 3 and 4 ) before I turn to a comparison of the results in Sect. 5 and some concluding remarks in Sect. 6. 


\section{The model}

The model in which the analysis will be performed is built on a few simplifying assumptions: there are only two homogeneous goods in the economy, medical care and a composite commodity, "consumption". As the need for medical services is stochastic and individuals are risk-averse, however, they are ex ante not interested in medical services per se but in health care insurance. The society consists of $n$ consumers (voters), and the amounts of the health insurance and consumption goods received by the $i$ th voter are denoted by $H_{i}$ and $x_{i}$, respectively. All consumers have the same additively separable preference function

$$
U_{i}=u\left(x_{i}\right)+\alpha_{i} \cdot v\left(H_{i}\right) \text { with } v^{\prime}, u^{\prime}>0, v^{\prime \prime}, u^{\prime \prime}<0,
$$

where the $U_{i}$ differ only in the taste parameter $\alpha_{i}$, which is a proxy for their relative preferences for health vis-à-vis other goods.

Besides their tastes, individuals also differ in their exogenously given market incomes $y_{i}$, and $Y$ denotes the average income. There are no taxes or transfers except the contributions to a social health insurance program which are levied as a strictly proportional income tax at the rate $t$. Thus nobody can opt out of the social health insurance ${ }^{3}$. Furthermore, as we are not interested in the effects of relative price changes, the prices of the two commodities can be normalized to unity.

Finally it is assumed that there is no altruism among individuals so that each voter behaves in a selfish way so as to maximize his own utility $U_{i}$.

\section{The case of strong rationing}

Here it is assumed that health services are provided exclusively through social health insurance. Therefore, each consumer receives the same amount $H$ of insurance coverage,

$$
H_{i}=H=t \cdot \frac{1}{n} \cdot \sum_{i=1}^{n} y_{i}=t \cdot Y
$$

and the budget constraint of the $i$ th consumer is given by

$$
x_{i}=(1-t) \cdot y_{i} .
$$

Inserting (2) and (3) into (1), we can express the $i$ th voter's utility as a function of the contribution rate $t$ :

$$
U_{i}(t)=u\left[(1-t) \cdot y_{i}\right]+\alpha_{i} \cdot v(t \cdot Y),
$$

and the optimal value for the $i$ th voter, $t_{i}^{*}$, is obtained implicitly from the firstorder condition:

$$
Y \cdot \alpha_{i} \cdot v^{\prime}\left(t_{i}^{*} \cdot Y\right)=y_{i} \cdot u^{\prime}\left[\left(1-t_{i}^{*}\right) \cdot y_{i}\right],
$$

since, due to (1), the sufficient second-order condition for a maximum of (4) 


$$
\frac{d^{2} U_{i}}{\mathrm{~d} t^{2}}=y_{i}^{2} \cdot u^{\prime \prime}\left[(1-t) \cdot y_{i}\right]+\alpha_{i} \cdot Y^{2} \cdot v^{\prime \prime}(t \cdot Y) \equiv D<0
$$

is fulfilled everywhere.

To determine how the desired value of the tax, $t_{i}^{*}$, varies with income $y_{i}$ and tastes, we totally differentiate Eq. (5) and obtain:

$$
\alpha_{i} \cdot Y^{2} \cdot v^{\prime \prime} \cdot \mathrm{d} t_{i}^{*}+Y \cdot v^{\prime} \cdot \mathrm{d} \alpha_{i}=\left\{u^{\prime}+y_{i} \cdot\left(1-t_{i}^{*}\right) \cdot u^{\prime \prime}\right\} \cdot \mathrm{d} y_{i}-y_{i}^{2} \cdot u^{\prime \prime} \cdot \mathrm{d} t_{i}^{*},
$$

and thus, invoking the implicit function theorem, one obtains the response of the optimal $t_{i}^{*}$ to isolated changes of the parameters $y_{i}$ and $\alpha_{i}$, respectively (with $D$ defined in Eq. (6)):

$$
\begin{aligned}
& \frac{\mathrm{d} t_{i}^{*}}{\mathrm{~d} \alpha_{i}}=-\frac{Y \cdot v^{\prime}}{D}>0 \\
& \frac{\mathrm{d} t_{i}^{*}}{\mathrm{~d} y_{i}}=\frac{u^{\prime}+\left(1-t_{i}^{*}\right) \cdot y_{i} \cdot u^{\prime \prime}}{D}=\frac{u^{\prime} \cdot\left(1-\eta_{u^{\prime}, x}\right)}{D} \gtreqless 0 \\
& <=>\eta_{u^{\prime}, x} \gtreqless 1,
\end{aligned}
$$

where

$$
\eta_{u^{\prime}, x}=-\frac{u^{\prime \prime}(x) \cdot x}{u^{\prime}(x)}
$$

measures the elasticity of marginal utility of the consumption good.

Not surprisingly, $t_{i}^{*}$ rises with increasing relative valuation of health services. Furthermore, given the preference parameter $\alpha_{i}$ the desired contribution rate $t_{i}^{*}$ rises (falls) unambiguously with increasing income $y_{i}$ if and only if the elasticity of marginal utility of consumption is larger (smaller) than 1.

It follows trivially that the median voter is the consumer with median income whenever all voters have identical tastes (identical $\alpha_{i}$ ). In any event there are two minorities the members of which do not get what they most prefer: in the "elastic case" $\left(\eta_{u^{\prime}, x}>1\right)$, people with above-median incomes would like to put more and all others would like to put less money into health care. In the "inelastic case" $\left(\eta_{u^{\prime}, x}<1\right)$, it is the other way around.

Moreover, when people differ in both tastes and incomes it is no longer certain that somebody with median income and median tastes must get his desired contribution rate elected even if the two exogenous parameters $y_{i}$ and $\alpha_{i}$ are distributed independently. This can be seen from (5) by fixing $t^{*}$ at the value desired by the median voter, $t_{m}^{*}$ and solving for $\alpha_{i}$ as a function of $y_{i}$ :

$$
\alpha_{i}^{*}\left(y_{i}\right)=\frac{y_{i} \cdot u^{\prime}\left[\left(1-t_{m}^{*}\right) \cdot y_{i}\right]}{Y \cdot v^{\prime}\left(t_{m}^{*} \cdot Y\right)},
$$

where it is obvious that $\alpha_{i}$ is not a linear function of $y_{i}{ }^{4}$, which proves the point that the graph of this function, i.e. the locus of all $\left(\alpha_{i}, y_{i}\right)$-combinations which separates the set of voters $i$ favoring a $t_{i}^{*}>t_{m}^{*}$ from the set favoring a $t_{i}^{*}<t_{m}^{*}$ does not have to pass through $\left(\alpha_{m}, y_{m}\right)$, where $m=(n+1) / 2$ (see Fig. 1). 


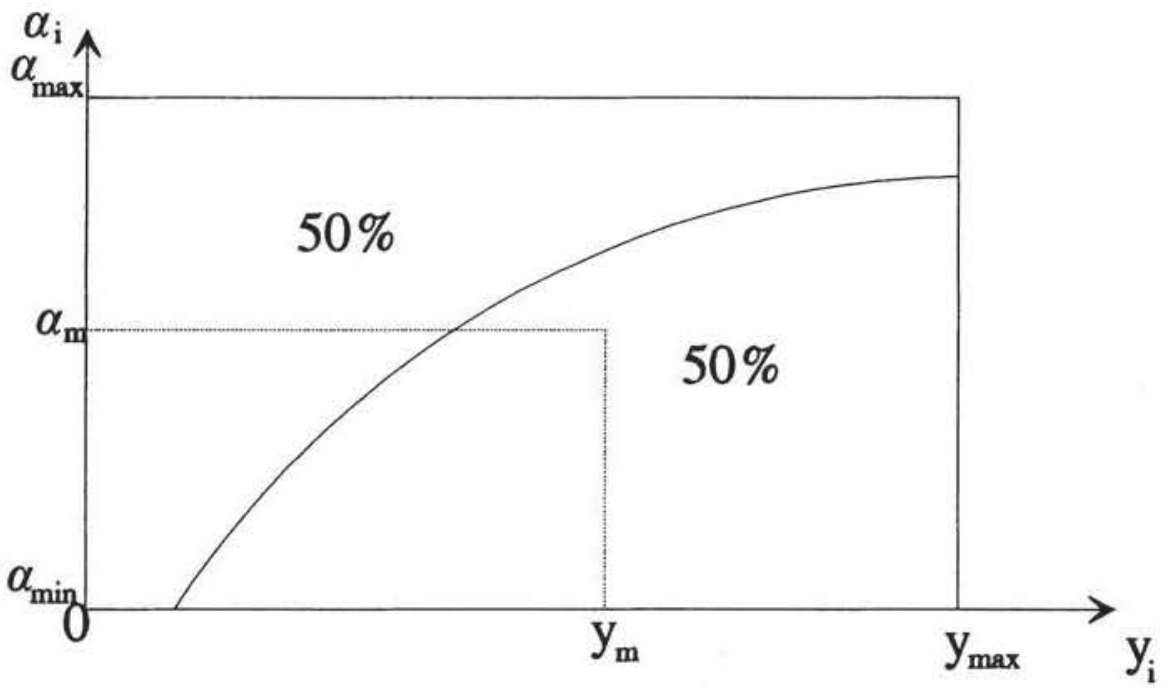

Fig. 1. Separating the voters according to their most preferred tax rate $t_{i}^{*}$

\section{The case of weak rationing}

We now turn to the case in which it is possible to buy supplementary health insurance coverage privately. The size of the supplementary demand is denoted by $h_{i}$ so that the total amount of health insurance coverage received by the $i$ th voter is

$$
H_{i}=t \cdot Y+h_{i} .
$$

Here each individual has to solve a two-stage optimization problem. On the lower level, for given contribution rate $t$, he has to determine the amount of his private supplementary coverage $h_{i}$. On the upper level, he has to vote for a contribution rate, taking into account the result of his lower-level decisions.

\subsection{Conditions for supplementary coverage}

As a point of reference we first derive the private demand for coverage of a consumer with income $y_{i}$ and tastes $\alpha_{i}$ under the assumption that a social health insurance program does not exist. For this case the maximand (1) becomes

$$
U_{i}\left(h_{i}\right)=u\left(y_{i}-h_{i}\right)+\alpha_{i} \cdot v\left(h_{i}\right),
$$

and the necessary first-order condition for a maximum of (12) is

$$
\alpha_{i} \cdot v^{\prime}\left(h_{i}\right)=u^{\prime}\left(y_{i}-h_{i}\right),
$$

and the total differential of (13) yields

$$
\frac{\mathrm{d} h_{i}}{\mathrm{~d} y_{i}}=\frac{u^{\prime \prime}}{u^{\prime \prime}+\alpha_{i} \cdot v^{\prime \prime}}>0 \text {, }
$$


i.e. for given tastes $\alpha_{i}$ the amount of insurance coverage privately demanded rises unambiguously with income.

In the general case, utility as a function of $h_{i}$ and $t$ becomes:

$$
U_{i}\left(h_{i}, t\right)=u\left[(1-t) \cdot y_{i}-h_{i}\right]+\alpha_{i} \cdot v\left(t \cdot Y+h_{i}\right),
$$

and the necessary first-order condition with respect to $h_{i}$ for given $t$ is, taking into account the non-negativity constraint on $h_{i}$ :

$$
\alpha_{i} \cdot v^{\prime}\left(t \cdot Y+h_{i}\right)-u^{\prime}\left[(1-t) \cdot y_{i}-h_{i}\right] \leq 0\left(=0 \text { if } h_{i}>0\right) .
$$

Therefore, a necessary and sufficient condition for a positive supplementary demand $\left(h_{i}>0\right)$ is:

$$
\alpha_{i} \cdot v^{\prime}(t \cdot Y)>u^{\prime}\left[(1-t) \cdot y_{i}\right] .
$$

\subsection{Determining the political equilibrium}

In the upper-level decision problem, the necessary first-order condition for the optimum contribution rate for the $i$ th individual is

$$
\frac{\partial U_{i}}{\partial t}=-y_{i} \cdot u^{\prime}\left[\left(1-t_{i}^{*}\right) \cdot y_{i}-h_{i}\right]+\alpha_{i} \cdot Y \cdot v^{\prime}\left[t_{i}^{*} \cdot Y+h_{i}\right] \leq 0\left(=0 \text { if } t_{i}^{*}>0\right),
$$

and the optimum value $t_{i}^{*}$ is zero if and only if

$$
y_{i} \cdot u^{\prime}\left[y_{i}-h_{i}\right] \geq \alpha_{i} \cdot Y \cdot v^{\prime}\left[h_{i}\right]
$$

with $h_{i}$ determined in (16), i.e.

$$
\alpha_{i} \cdot v^{\prime}\left[h_{i}\right]=u^{\prime}\left[y_{i}-h_{i}\right],
$$

so that (19) is equivalent to

$$
y_{i} \geq Y .
$$

Clearly, in a system of weak rationing no selfish individual with income at or above the average will vote for a positive contribution rate since for these individuals the effective price per unit of health insurance coverage in the public system exceeds the one in the private system. (21) also shows that there will still be a majority for a positive size of the public system if, as is usually the case, median income $y_{m}$ falls short of mean income $Y$.

To determine the contribution rate in a political equilibrium, $t_{m}^{*}$, we note that the optimality condition (18) must be fulfilled as an equality for an individual $i$ with $h_{i}=0$, whereas for $(n+1) / 2$ individuals with $h_{i}=0$ the same condition must be fulfilled as an inequality:

$$
\begin{aligned}
& \alpha_{i} \cdot Y \cdot v^{\prime}[t \cdot Y] \geq y_{i} \cdot u^{\prime}\left[(1-t) \cdot y_{i}\right] \text { for all } i \in S \text { with } \# S=(n+1) / 2 \\
& \alpha_{i} \cdot Y \cdot v^{\prime}[t \cdot Y]=y_{i} \cdot u^{\prime}\left[(1-t) \cdot y_{i}\right] \text { for one } i \text {. }
\end{aligned}
$$


Determining the median voter is easy if the function $u^{\prime}(\cdot)$ is separable in the following way:

$$
u^{\prime}\left[(1-t) \cdot y_{i}\right] \equiv \Phi(t) \cdot f\left(y_{i}\right) \text { with } \Phi^{\prime}(t)>0 .
$$

In this case the individuals can be arranged according to the variable

$$
b_{i}=\frac{y_{i} \cdot f\left(y_{i}\right)}{\alpha_{i}},
$$

and for the $(n+1) / 2$ th-smallest value, $b_{m}$, the value of $t$ which solves the equation

$$
b_{m}=\frac{Y \cdot v^{\prime}[t \cdot Y]}{\Phi(t)}
$$

is the median contribution rate $t_{m}^{*}$. All individuals for which (22) is fulfilled as a strict inequality at this value of $t$ would prefer a higher value, $t_{i}^{*}$.

More specific statements on the equilibrium contribution rate $t_{m}^{*}$ and on the structure of the supplementary private purchases of health insurance coverage can be made if more restrictive assumptions on the distribution of the taste parameter $\alpha_{i}$ are made.

In the simplest case, all individuals have identical tastes, so that we can set all $\alpha_{i}=1$. Then inserting (23) into (24) yields

$$
b_{i}=\frac{y_{i} \cdot u^{\prime}\left[(1-t) \cdot y_{i}\right]}{\Phi(t)},
$$

and the first derivative of $b_{i}$ with respect to $y_{i}$,

$$
\frac{\mathrm{d} b_{i}}{\mathrm{~d} y_{i}}=\frac{u^{\prime}\left[(1-t) \cdot y_{i}\right]}{\phi(t)} \cdot\left(1-\eta_{u^{\prime}, x}\right) \gtreqless 0 \text { as } \eta_{u^{\prime}, x} \lesseqgtr 1 \text {. }
$$

Thus, for identical tastes we can distinguish the three following subcases (which are illustrated in Fig. 2a, b, c):

a) the elasticity of marginal utility of consumption is below unity. Then the value of $b_{i}$ rises with the income $y_{i}$, and hence the desired contribution rate unambiguously falls with income. This implies that the median voter is the voter with median income, and all voters with smaller income would prefer a larger size of the social health insurance scheme;

b) the elasticity of marginal utility of consumption is unity, i.e. utility is logarithmic. Then everybody has the same $b_{i}$, and thus there is no conflict of interest among below-average-income voters;

c) the elasticity of marginal utility of consumption is greater than unity. Then the value of $b_{i}$ falls with income $y_{i}$, and hence among voters with below-average incomes the desired contribution rate rises with income. The median voter is thus close to the left-hand tail of the income distribution so that exactly 50 per cent of the electorate have incomes between his and the mean value. Let 
a) $\eta_{u^{\prime}, x}<1$

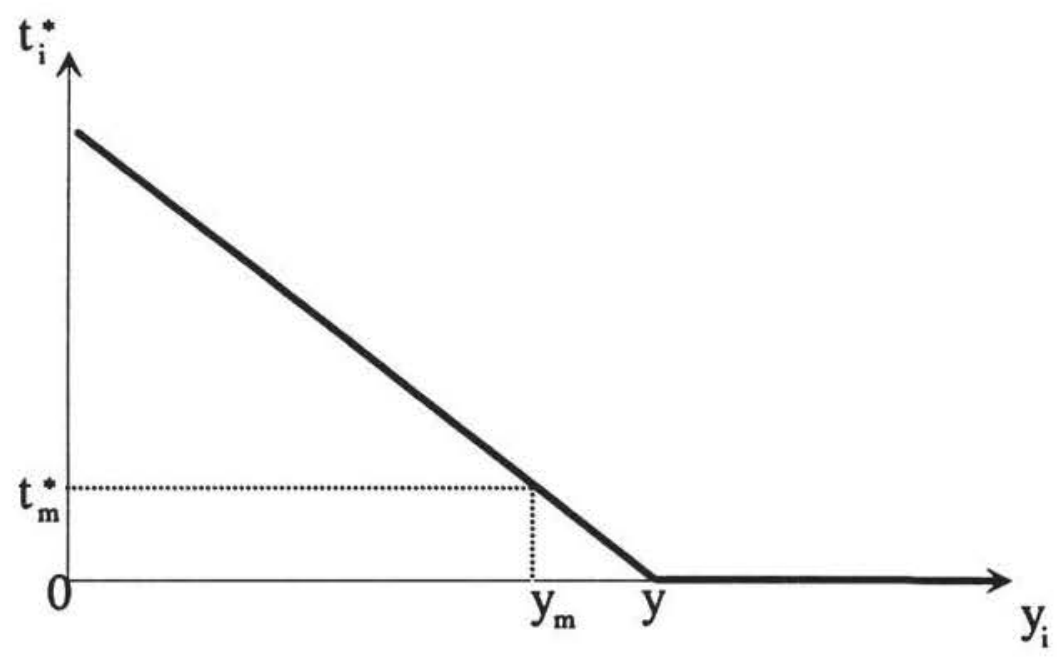

b) $\eta_{u^{\prime}, \mathrm{x}}=1$

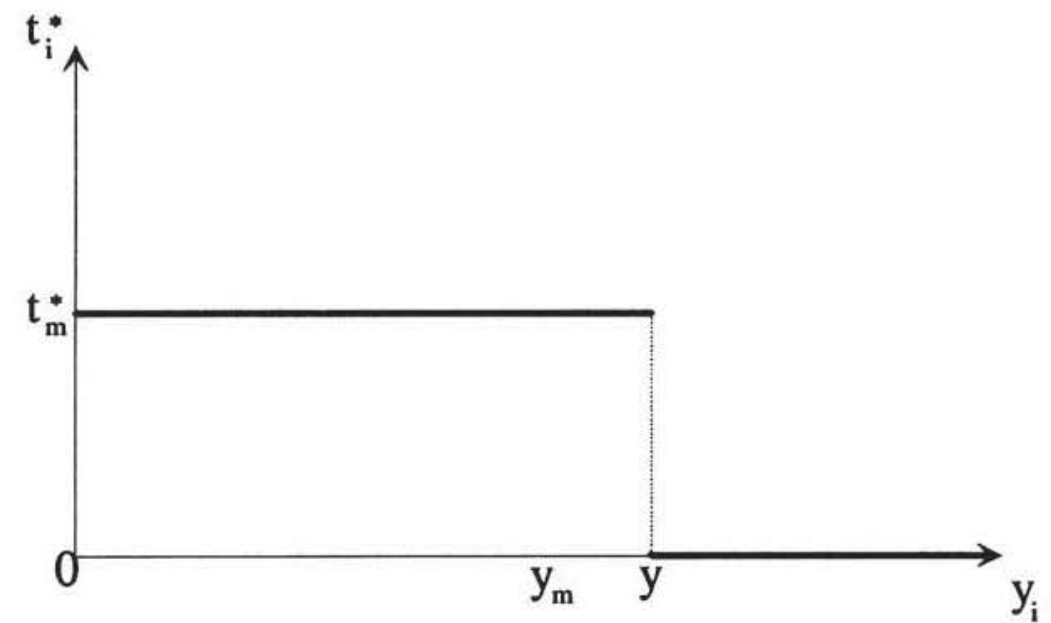

Fig. 2a-c. The desired contribution rate to social health insurance as a function of income

us denote his income by $y_{\text {low }}$. All voters in this intermediate range $\left[y_{\text {low }}, Y\right]$ would prefer a larger size of the social health insurance scheme.

\subsection{The purchase of supplementary coverage}

Next we shall determine the structure of the private demand for supplementary health insurance. We notice first that the set of private demanders is by no means identical to the set of voters who would have preferred a larger size of the public health insurance scheme because the prices of the two goods "public" and 
c) $\eta_{u^{\prime}, \mathrm{x}}>1$

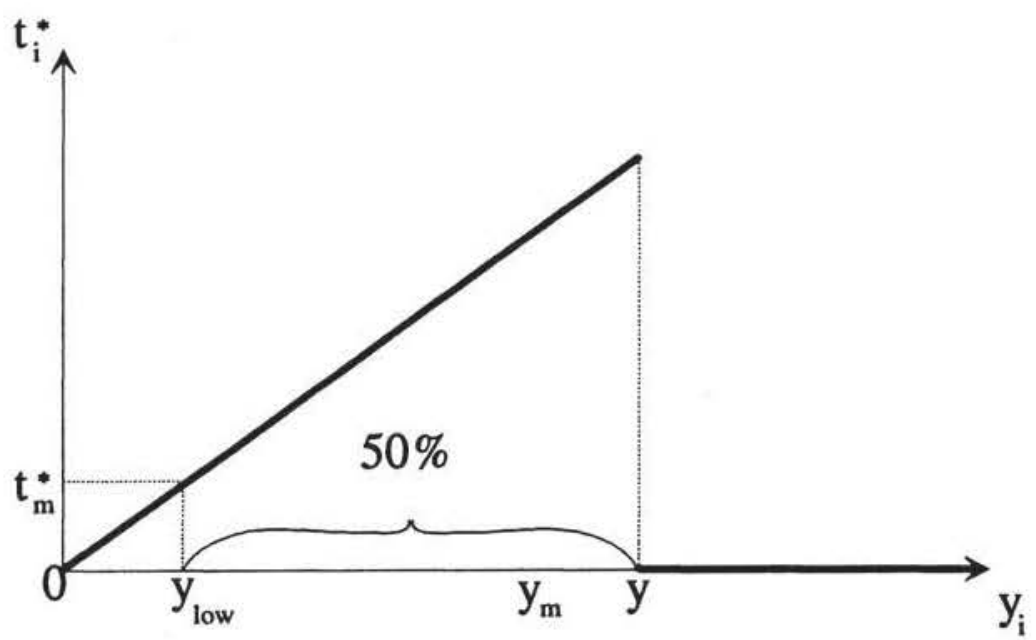

Fig. 2c

"private health insurance coverage" differ across income groups. Formally, the condition for the desire of higher-than-equilibrium contribution rate, (22), is equivalent to

$$
V_{i}\left(y_{i}\right) \equiv \alpha_{i} \cdot v^{\prime}\left[t_{m}^{*} \cdot Y\right]-u^{\prime}\left[\left(1-t_{m}^{*}\right) \cdot y_{i}\right] \cdot \frac{y_{i}}{Y}>0,
$$

whereas from (17) the condition for the purchase of supplementary health insurance at this size of social health insurance is

$$
P_{i}\left(y_{i}\right) \equiv \alpha_{i} \cdot v^{\prime}\left[t_{m}^{*} \cdot Y\right]-u^{\prime}\left[\left(1-t_{m}^{*}\right) \cdot y_{i}\right]>0,
$$

and it is immediately clear that the two variables $V_{i}$ (for "voting behavior") and $P_{i}$ (for "purchasing behavior") are related to each other by the condition

$$
P_{i}\left(y_{i}\right) \gtreqless V_{i}\left(y_{i}\right) \text { whenever } y_{i} \gtreqless Y \text {. }
$$

Note further that $P_{i}$ is continuous and always increasing in $y_{i}$ (due to $u^{\prime \prime}<0$ ).

Using these definitions we can now analyze the purchasing behavior of individuals in the political equilibrium using the results of the previous section:

a) In the case of $\eta_{u^{\prime}, x}<1$, the variable $V_{i}$ is

- decreasing in $y_{i}$,

- equal to zero for median income $y_{m}$ and

- larger than zero for $y_{i}<y_{m}$.

Consequently, $P_{i}$ is smaller than 0 for average income $\left(y_{i}=Y\right)$. Thus, because it is increasing in $y_{i}$, there exists a lower bound $y_{a}^{* *}\left(y_{a}^{* *}>Y\right)$ for the purchase of supplementary private coverage, and only the (very) rich, if anybody, demand such coverage. 
b) In the case of logarithmic utility $\left(\eta_{u^{\prime}, x}=1\right), V_{i}$ is equal to zero for all $y_{i}<Y$, and thus $P_{i}$ is

- smaller than zero for $y_{i}<Y$ and

- larger than zero for $y_{i}>Y$.

Again there is a lower income bound $y_{b}^{* *}$ for the purchase of private coverage, but this time $y_{b}^{* *}$ is equal to mean income $Y$.

c) In the case of $\eta_{u^{\prime}, x}>1, V_{i}$ is

- increasing in $y_{i}$ and

- positive for all $y_{i}$ in the interval $\left[y_{\text {low }}, Y\right]$.

Consequently, $P_{i}$ is smaller than zero for $y_{i}=y_{\text {low }}$ and larger than zero for $y_{i}=Y$, and thus there must again be a lower limit $y_{c}^{* *}$ with $y_{\text {low }}<y_{c}^{* *}<Y$ such that supplementary coverage is purchased by all consumers with $y_{i}>y_{c}^{* *}$.

We can thus conclude that for identical tastes total coverage $H_{i}$ is always a (weakly) increasing function of pre-tax income $y_{i}$. In any event there exists a lower income bound $y^{* *}$ (which can be smaller, equal to or larger than average income $Y$ ) such that

- all households $i$ with $y_{i} \leq y^{* *}$ just have social health insurance coverage, and

- all households $i$ with $y_{i}>y^{* *}$ buy supplementary private health insurance coverage.

\section{Comparison of the results}

\subsection{The elasticity of marginal utility of consumption}

In the derivation of the results in Sects. 3 and 4, it turned out that an important factor is the elasticity of marginal utility of consumption. Thus it would be desirable to check the empirical value of this parameter. Here, direct evidence is not available, but we can invoke some theoretical considerations and combine them with indirect empirical evidence.

First suppose that there were no government involvement in the financing of health insurance so that the insured would have to bear the full cost. In this case it is easy to show that the first-order condition for the individual utility maximization problem is (dropping the subscript $i$ for convenience)

$$
u^{\prime}(x)=\alpha \cdot v^{\prime}(H) .
$$

Furthermore, if the household income $y$ rises and relative prices remain constant, then the relative shares of the two types of consumption behave according to the following equation:

$$
\frac{\mathrm{d}(H / x)}{\mathrm{d} y}=\frac{x \cdot u^{\prime \prime}-\alpha \cdot H \cdot v^{\prime \prime}}{x^{2} \cdot D}=\frac{-u^{\prime} \cdot \eta_{u^{\prime}, x}+\alpha \cdot v^{\prime} \cdot \eta_{v^{\prime}, H}}{x^{2} \cdot D},
$$

and thus, due to (31) and $D<0$ the relative share of health care expenditures, $H / x$, rises with household income if

$$
\eta_{u^{\prime}, x}>\eta_{v^{\prime}, H},
$$


i.e. if the elasticity of marginal utility of (other) consumption exceeds that of health services.

An empirical cross-section analysis for countries (Newhouse 1977) has shown that with increasing per-capita income the share of health care expenditures in GNP rises significantly. This result is applicable for the model presented in this section since a nation as a whole has to bear the entire cost of its health care consumption. Moreover, since it was a cross-section, the finding can not be due to medical progress in the wake of economic growth.

Therefore, we can conclude that if the constant-elasticity-of-marginal-utility function presented in (1) approximately describes the preferences of people towards health care and other consumption, then inequality (33) appears to be fulfilled. This alone does not yet prove that the left-hand side of (33) is bigger than one. Observe, further, that with the type of utility function considered,

$$
v(H)=\frac{1}{1-\gamma} \cdot H^{1-\gamma}
$$

where $\gamma$ measures the elasticity of marginal utility, $\eta_{v^{\prime}, H}$, utility is bounded (unbounded) if $\gamma$ exceeds (falls short of) unity. Since it is plausible that even if health care consumption rises beyond all bounds, utility from it remains limited, the right-hand side of (33) is probably bigger than one, and so is the left-hand side.

\subsection{Consequences for the two types of rationing}

We are now able to compare the results of the two preceding sections with respect to two questions:

1. Which of the two rationing systems leads to a higher total medical care consumption?

2. Which of the two rationing systems leads to higher utility level of the respective income groups? Is it even possible to make a Pareto comparison?

Both questions are easy to answer for identical preferences and the case of relatively inelastic marginal utility of consumption $\left(\eta_{u^{\prime}, x} \leq 1\right)$. In this case, which is the less plausible one according to Sect. 5.1, it is always the median income earner who gets his first preference elected, and thus - as I assume throughout $y_{m}<Y$ - the size of the public health insurance coverage is the same in both rationing systems. However, since the rich will buy additional coverage privately if this is possible, weak rationing leads both to a higher total health care consumption and to a higher welfare in the Paretian sense.

Therefore, suppose that the type of rationing system itself were up to a vote and voters had perfect foresight of the political equilibrium in either case, their self-interest would clearly dictate to opt for a weak rationing system.

Things are less clear-cut in the more plausible opposite case $\left(\eta_{u^{\prime}, x}>1\right)$. While it is obvious that the availability of private coverage reduces the size of the public system in this case, it is not clear a priori whether the private purchases compensate for this difference. However, the normative question has a definite answer. The weak rationing system clearly benefits at least two groups:

- those who earn very little $\left(y_{i}<y_{\text {low }}\right)$ and those with incomes only slightly above $y_{\text {low }}$ get a size of the public system at or much closer to their optimum than under strong rationing; 
- all voters with above-average income also get a total coverage corresponding to their own preferences and at a lower total price than under strong rationing.

On the other hand, the median income earner gets exactly his optimal (total) coverage in both systems, but the price is higher for him under weak rationing. Therefore, neither system Pareto-dominates the other.

However, the result of a voting on the type of rationing system as such is determinate since the two groups mentioned above comprise together more than $50 \%$ of the electorate $y_{\text {low }}$ was defined in Sect. 4.2 by the provision that exactly $50 \%$ have incomes either below $y_{\text {low }}$ or above the average income, $Y$. See also Fig. $2 \mathrm{c}$ ).

\section{Concluding remarks}

The present paper can be regarded as a very preliminary consideration of the likely results of voting on the size of a public health insurance system in a direct democracy. The significance of the findings is limited by the many restrictive assumptions employed such as the absence of altruism. It should be emphasized that definite results have been shown only for the case of identical (relative) preferences for health and other consumption and the special class of constantelasticity-of-marginal-utility-of-consumption functions.

Finally it should be mentioned that the framework is entirely static so that the intergenerational transfer effects involved in the existing public health insurance systems and their impact on the political decision-making (which were analyzed in Breyer and v. d. Schulenburg 1987) were completely neglected here.

\section{Endnotes}

1 Counterarguments against this assumption are discussed in Breyer and Kliemt 1994.

2 This assumption is approximately satisfied in the German sickness fund system in which only the top $20 \%$ of the income distribution are allowed to opt out and contributions are proportional to labor income up to a ceiling.

${ }^{3}$ For constant-elasticity-of-marginal-utility functions the function $\alpha_{i}^{*}\left(y_{i}\right)$ is increasing and concave if $\eta<1$ but decreasing and convex if $\eta>1$.

\section{References}

Breyer F, Kliemt H (1994) Lebensverlängernde medizinische Leistungen als Clubgüter? - Ein Beitrag zum Thema "Rationierung im Gesundheitswesen“. In: Homann K (ed) Wirtschaftsethische Perspektiven I: Theorie - Ordnungsfragen - Internationale Institutionen. Duncker \& Humblot, Berlin, pp $131-158$

Breyer F, Schulenburg J-M Graf v. d. (1987) Family structure and intergenerational transfers in social health insurance: A public choice model. In: Pethig R, Schlieper U (eds) Efficiency, institutions, and economic policy. Springer, Berlin Heidelberg New York, pp 63-80

Newhouse JP (1977) Medical-care expenditures: A cross-national survey. J Hum Res 12:115-125 